\title{
Multilinguales
}

Multilinguales

14 | 2020

Enseignement universitaire et professionnalisation : enjeux, questionnements et défis socio-économiques

\section{L'altérité vue par de jeunes apprenants de langue}

Othernes seen by young language learners

Hidaya Djouder et Billel Ouhaibia

\section{(2) OpenEdition}

Journals

Édition électronique

URL : https://journals.openedition.org/multilinguales/5663

DOI : $10.4000 /$ multilinguales.5663

ISSN : 2335-1853

Éditeur

Université Abderrahmane Mira - Bejaia

Référence électronique

Hidaya Djouder et Billel Ouhaibia, "L'altérité vue par de jeunes apprenants de langue », Multilinguales [En ligne], 14 | 2020, mis en ligne le 10 décembre 2020, consulté le 02 juillet 2021. URL : http://

journals.openedition.org/multilinguales/5663; DOI : https://doi.org/10.4000/multilinguales.5663

Ce document a été généré automatiquement le 2 juillet 2021.

\section{(c) (1) $\Theta(9$}

Multilinguales est mise à disposition selon les termes de la Licence Creative Commons Attribution -

Pas d'Utilisation Commerciale - Pas de Modification 4.0 International 


\title{
L'altérité vue par de jeunes apprenants de langue
}

\author{
Othernes seen by young language learners
}

Hidaya Djouder et Billel Ouhaibia

1 Le troisième millénaire est marqué par des bouleversements idéologiques interprétés dans des notions telles que mondialisation de l'information, internationalisation, plurilinguisme, interculturalisme, etc. La didactique des langues, secouée, elle aussi, par la vague de «l'inter» (interlinguistique, intersocialisation, interculturel, interaction), se préoccupe actuellement de l'enseignement/apprentissage des langues en interaction et non en juxtaposition. La didactique de contact tente de valoriser l'identité de l'autre dans le but de s'ouvrir à l'altérité et de respecter la différence, mais également afin d'inciter à la prise de conscience de son identité dans la diversité.

Les représentations, qui constituent un élément important dans la construction des connaissances nouvelles et le développement d'attitudes positives à l'égard de l'autre, sont désormais prises en compte dans l'enseignement de langue. Considérées comme un système de référence, les représentations renvoient également à un processus dynamique et évolutif.

En ce sens, ne peut-on pas s'interroger sur les représentations de l'autre dans le contexte scolaire algérien? Étant donné que des études locales ont révélé comment les grands adolescents et les adultes se représentent-ils l'altérité, nous nous préoccuperons ici de la façon dont les jeunes apprenants de langue se la représente-t-ils. L'objectif de notre travail tente d'explorer les opinions des élèves afin de mettre en œuvre un enseignement de l'altérité qui permet de réguler le rapport à l'autre.

2 Dans ce qui suit, nous essaierons dans un premier temps d'expliciter la priorité d'une éducation à l'altérité en continuité et/ou en rupture avec une éducation interculturelle. Dans un deuxième temps, nous aborderons l'importance des représentations dans la prise de conscience de l'autre et de soi, en particulier en contexte algérien. Enfin dans un dernier temps, nous ferons part d'une analyse thématique des représentations suite à une expérience menée auprès d'élèves de $5^{\mathrm{e}}$ année primaire. 


\section{De l'interculturel vers le rapport à l'altérité}

3 L'éducation interculturelle est basée sur la similitude et la différence des cultures dans le but de développer une compétence interculturelle jugée indispensable dans un monde mondialisé. Toutefois, cette approche interculturelle est prise un peu à la légère, en accentuant les similitudes ou exagérant les différences. Selon Lipiansky (1999:155), la formation interculturelle reposerait sur un principe "dissociatif», une communication fondée sur la différence, au lieu d'un principe « associatif » basé sur la similitude. De même, l'inter n'est pas réellement pris en compte. Les cultures visées ne sont pas en interaction. L'intégration de l'inter exige le développement de pratiques " métissées », en abandonnant certaines références et certains modèles anciens tels que "le monolingue natif», sur lequel on mesurerait la qualité des apprentissages. (Blanchet, 2014).

4 En contexte algérien, les travaux de chercheurs témoignent d'une volonté interculturelle. Selon Hamidou (2007: 35), l'éducation interculturelle permet de s'ouvrir à l'autre et à délimiter son identité culturelle. Gaouaou (2009:215) pense également que l'Algérie, marquée par sa diversité linguistique et culturelle adopterait bien l'éducation interculturelle afin d'inscrire la société dans la «matrice de la modernité ». Abdelhamid et Khadraoui (2009: 40) réfléchissent à un dépassement de sentiment de suprématie identitaire, considéré comme source de malentendus et de conflits. Selon eux, un "Bio-Dialogue $»^{1}$ conduit à une "Bio-Culture ${ }^{2}$ car les interactions profondes et diverses deviennent condition de vie, dont l'objet est d'humaniser la mondialisation.

Dakhia (2008: 147) s'interroge sur ce « désir d'interculturalité »: «(...) n'est-ce pas un autre idéal humain, une autre mode politique de gestion des consciences dont il est réellement question?». D'autant plus qu'interculturel porte en son sein le terme de culture. Un terme contourné dans le discours de la didactique des langues car trop marqué idéologiquement. D'où l'évolution d'une éducation culturelle à celle d'interculturelle. Cette dernière est, semble-t-il, en train d'évoluer à son tour vers une éducation encore plus englobante, caractérisée par des formes diverses.

Ce vouloir interculturel est bel et bien présent. Les directives officielles pour l'élaboration des programmes scolaires mettent même en avant la dimension culturelle/interculturelle, et ce depuis la réforme pédagogique de 2003. Cependant, l'enquête de terrain montre que l'éducation culturelle/interculturelle " (...) n'est pas suivie d'effets dans les documents des programmes officiels; elle disparaît complètement dans les manuels de français du secondaire ». (Khebas, Malika, citée par Demorgon, $2010: 235$ ).

5 La résistance face à l'intégration de l'aspect culturel dans l'enseignement de langue, et la réticence à la déculturation/acculturation sont exprimées à travers une survalorisation identitaire qui accentue le sentiment ego-ethnocentrique. Les concepteurs des programmes scolaires, même s'ils déclarent vouloir s'ouvrir à la diversité linguistique et culturelle intranationale et internationale, ne sont pas pour autant prêts à intégrer la culture française (dans toutes ces dimensions) dans les programmes d'enseignement, quoique cette première fasse partie du paysage linguistique et culturel de la société algérienne.

$\mathrm{Au}$ fait de la complexité de la situation d'intégration et le besoin d'adaptation des méthodologies apportées au contexte algérien, nous envisageons un enseignement/ 
apprentissage du français dans une dimension plurielle à travers l'expérience diversifiée de l'altérité. C'est-à-dire, enseigner la langue française en ayant recours à différentes formes de l'altérité (intégrer d'autres langues, d'autres cultures, d'autres modes), en plus de la culture identitaire et pas uniquement l'intégration de la culture de la langue cible.

\section{L'éducation à l'altérité}

6 L'altérité, notion à la mode, s'invite dans le champ de recherche de plusieurs disciplines (philosophie, psychologie sociale, sciences de l'éducation, didactique) où elle prend de l'ampleur. Hérité du latin alter (ce qui est autre, distinct, séparé) et du grec to heteron (ce qui est autre que, différent de), le terme altérité a pour origines l'antiquité grécolatine (Briançon, 2019 : 25/27).

Jodelet (2005: 10) associe l'altérité à la notion d'exclusion, et la positionne à deux niveaux. L'altérité du dehors, à caractère " lointain » voire " exotique ", renvoie aux pays, peuples et groupes situés dans un espace et/ou un temps distant. L'altérité du dedans concerne toutes les distinctions à l'intérieur d'un même ensemble social ou culturel. Elle se rapporte à une différence qui peut être d'ordre physique ou corporel, du registre des mœurs, ou par rapport à une appartenance de groupe.

7 En sciences de l'Éducation, l'altérité fait également objet de réflexion. Elle est envisagée sous des aspects différents (relationnelle, réflexive et épistémologique). Muriel Briançon (2012:19/20) distingue trois formes complémentaires d'altérité : extérieure, intérieure et épistémologique qu'elle unifie dans un seul concept celui d'«Altérité enseignante ». La même auteure interroge la didactique sur quel savoir didactique pourrait se rapprocher l'altérité, et invite les didacticiens à s'interroger voire à développer une didactique de l'altérité ${ }^{3}$.

Ses questions ont fait échos. L'altérité qui intéresse également les didacticiens est rapprochée au "savoir aborder, traiter l'autre, le différent, le nouveau " du CECR (2001), dans un cas particulier : le «savoir apprendre ». L'altérité est définie par Coste et Cavalli (2015: 19) comme: «(..) une construction psychologique qui survient lorsqu'une différence de quelque sorte est perçue et rendue saillante par la situation et/ou par les processus psychologiques de l'individu.». De plus, l'autre (perçu comme nouveau, différent) ne peut se restreindre à un autrui humain selon les mêmes auteurs, il « (...) peut être un individu, un groupe, une culture, mais aussi une connaissance à acquérir, une œuvre à découvrir ».

8 Pour Beacco (2018), la disponibilité à l'altérité ne s'acquiert pas selon des démarches formatives que s'il s'agissait d'un savoir-faire ou d'un savoir-être. L'altérité est plutôt rapprochée au "savoir-vivre ensemble", mais, selon lui, il est très impropre de considérer ce dernier savoir comme une "compétence ». Il est question de manières d'agir ou de comportements commandés par les valeurs que chacun s'est appropriées. L'adhésion à des valeurs dépend de la conscience de soi et du monde. Ainsi le précise Beacco $(2018: 100)$ :

Une éducation à l'altérité réduite à faire acquérir des comportements « corrects " au regard de standards définissant le «bon citoyen » ou la personne « ouverte à l'altérité » risque d'être superficielle, si l'on n'a pas, en même temps, suscité une adhésion véritable aux valeurs qui en sont le fondement.

La notion de valeur apparaît dans le nouveau programme du français de l'enseignement primaire en Algérie, dans lequel les valeurs identitaires, intellectuelles et esthétiques 
devraient être prises en charge. Les valeurs concernent le «(..) domaine de la formation de la personnalité, au même titre que les autres disciplines ». (Commission Nationale des Programmes, $2016: 4$ ). De ce point de vue, nous pouvons nous interroger si ces valeurs correspondent-elles aux standards du vivre ensemble et de l'ouverture sur l'autre, et nous demander comment les mettre en pratique en classe de langue.

Revenons à Beacco, pour qui l'enseignant de langue doit intervenir sur un ensemble d'attitudes : la curiosité, la bienveillance, l'ouverture à l'altérité, l'attitude critique. L'objectif est de susciter la curiosité et l'ouverture d'esprit des apprenants envers l'altérité et non de présenter une vision positive ou négative du pays et du peuple étranger (Gaouaou, 2009: 214). La langue, la culture et l'altérité représente un «triptyque » selon lequel des renouvellements méthodologiques ont été apportés dans l'enseignement des langues, ainsi que dans la connaissance des cultures (Merazga, Rachida, 2015 : 85). Ce qui amène à une clarification terminologique qui s'impose, en effet, Beacoo (2018: 14) préfère le terme «rapport à l'altérité" à celui d'« interculturel », du fait que celui-là est jugé plus neutre relativement aux débats sur la nature de l'interculturalité, et est centré sur l'altérité par rapport à soi.

\section{Le poids des représentations dans l'enseignement des langues}

10 Utilisée par des disciplines différentes, la notion de représentation se révèle très complexe. Les représentations sociales, abordées dans le champ de la psychologie sociale, sont définies ainsi (Jodelet, 1984, citée par Sallaberry, Jean-Claude, 1996: 23-24) :

Le concept de représentation sociale désigne une forme de connaissance spécifique, le savoir de sens commun, dont les contenus manifestent l'opération de processus génératifs et fonctionnels socialement marqués. Plus largement, il désigne une forme de pensée sociale. Les représentations sociales sont des modalités de pensée pratique orientées vers la communication, la compréhension et la maîtrise de l'environnement social, matériel et idéal.

Cela dit, la représentation sociale est considérée comme un système de référence, un ensemble de savoirs acceptés et partagés, permettant l'interprétation et la classification. En même temps, l'élaboration des représentations sociales repose sur une opération de processus établie par un sujet dans la construction de la réalité sociale. La représentation sociale renvoie donc, à la fois, à un produit et à un processus.

En sciences de la cognition, la théorie des représentations se rapporte à l'articulation du niveau logique individuel et le niveau logique collectif. À ce sujet, Sallaberry (1996 : 24-25) explique le phénomène de représentation comme suit :

C'est en échangeons les représentations que nous interagissons. Une telle description semble se situer exclusivement à un niveau "extérieur", extrapsychique; or il ne faut pas perdre de vue la dynamique intérieur/extérieur. $\mathrm{Au}$ niveau intrapsychique, l'hypothèse postule donc l'existence de «zones » en interaction, à l'intérieur de la psyché. Nous obtenons un concept (...) susceptible de "fonctionner" à "l'intérieur comme à «l'extérieur» de la psyché; donc susceptible aussi de fonctionner dans la dynamique intérieur/extérieur.

Ce qui nous permet de penser que cette dynamique des représentations est susceptible d'aider les sujets à changer en modifiant leurs représentations par l'interaction au 
niveau extérieur (importante dans la construction des rapports sociaux), mais aussi par l'interaction au niveau intérieur, c'est-à-dire interagir avec soi-même.

D'après Lipiansky $(1992,1999$ : 147), la perception de l'étranger implique trois mécanismes : un effet de contraste, un effet de stéréotypie et un effet d'assimilation. Pour lui, l'ethnocentrisme est un besoin naturel et premier face à l'altérité, et est corrélatif des mécanismes de distinction qui séparent le familier et l'inconnu, les proches et les étrangers.

Pour ne pas rester dans un état de distanciation qui peut mener à une situation d'aliénation extrême, la rencontre interculturelle (ou la rencontre de l'altérité) nécessite un milieu de sympathie ; l'établissement d'un espace de miroitements ; enfin, un besoin collectif de complémentarité. (Kordes, 1994, 1999 : 172).

13 Catherine Boyer (2000: 151) explique que les conceptions (une forme possible de la représentation) apportent des renseignements sur la construction des connaissances à travers les énoncés et les textes émis par le sujet. « (...) Lorsque l'on se place en début d'apprentissage, nous appelons ces conceptions: représentations initiales. Nous entendons ainsi pointer ce déjà-là intellectuel construit par les enfants, à l'école et hors de l'école, et dont nous savons l'importance dans la construction des connaissances ».

\section{L'analyse des représentations en contexte algérien}

Les analyses des représentations, dont la plupart concernent les lycéens et les étudiants, montrent un rapport limité, voire un rejet de l'altérité et manifestation d'un sentiment ethnocentrique (Djedid \& Aouadi, 2015 ; Djedid, 2016 ; Hamidou, 2014). Aux yeux des apprenants du secondaire (de la région ouest de l'Algérie), l'image de la France est celle d'un "pays de Cocagne ». Quant au Français, il est perçu comme raciste, mais instruit, cultivé, travailleur, sérieux, moderne, poli. (Hamidou, 2014 : 126-127). Pour ce qui est des étudiants algériens (de l'université d'El-Tarf), leurs réponses laissent paraître une représentation négative qui correspond à une altérité radicale où l'autre est représenté comme un étranger. L'autre est placé dans un espace distant qui renvoie à ce que Jodelet appelle « l'altérité du dehors ». (Djedid, \& Aouadi, $2015: 83$ ).

Afin de prévenir l'installation des représentations négatives de l'altérité, nous pensons qu'il est judicieux de revoir les apprentissages fondamentaux, et de se baser sur une dimension relationnelle au même titre que lire, écrire et compter. En effet, la langue est actuellement conçue comme un acte relationnel qui engage la personne de l'intérieur d'elle-même.

Selon Piaget (1921:29), la pensée de l'enfant est plus proche d'un ensemble d'attitudes ressortissant à la fois de l'action et de la rêverie que de la pensée. Se refuser d'admettre a priori l'absolue conformité des représentations de l'enfant avec celles de son entourage est légitime pour Piaget. « Les mots (...) sont les mêmes chez l'enfant et chez nous, mais ils ont des sens différents, plus large ou moins large suivant les cas. ».

À partir de nouvelles orientations méthodologiques postulées notamment par Coste et Cavalli (2015), Beacco (2018), nous hypothésons qu'exposer les enfants à une altérité plurielle en interaction avec l'identité, permet une disposition favorable à l'égard de l'autre. 


\section{La révélation des mots chez le jeune apprenant}

Les représentations initiales des apprenants permettent d'avoir une idée sur leur vision du monde, et par-delà à concevoir des activités permettant l'évolution de leurs conceptions de l'altérité, dans un espace d'échange et d'interaction.

En effet, l'enquête de classe que nous exposons vise deux objectifs. L'un concerne l'émergence des représentations des apprenants âgés entre 9 et 11 ans, scolarisés en $5^{\mathrm{e}}$ AP pour constituer des activités sur la thématique de l'altérité à partir du déjà-là. L'autre est d'exposer les élèves en question à différentes formes d'altérité : linguistique et culturelle; conceptuelle ; relationnelle pour les amener vers des formes de curiosité, de bienveillance, d'ouverture à l'autre. Un ensemble d'attitudes sur lequel l'enseignant doit intervenir selon Beacco.

Pour Moscovici, le contenu d'une représentation sociale est constitué de trois types d'éléments: les opinions, les attitudes et les stéréotypes (Negura, 2006:3). Dans le présent travail, nous nous intéressons aux opinions des élèves sur ce que Briançon appelle l'«altérité extérieure » qui se rapporte à l'altérité d'autrui, nos rapports à autrui oscillant entre conflit et rencontre pouvant gérer mystère, incompréhension et désir.

Dans l'analyse des représentations des élèves, nous avons opté pour une analyse thématique, se référant au travail de Negura (2006), afin de dégager des unités sémantiques de base. Les opinions des élèves sont collectées à partir d'une activité Remue-méninges (brainstorming) présentant les enfants du monde.

Figure 1 : Remue-méninges

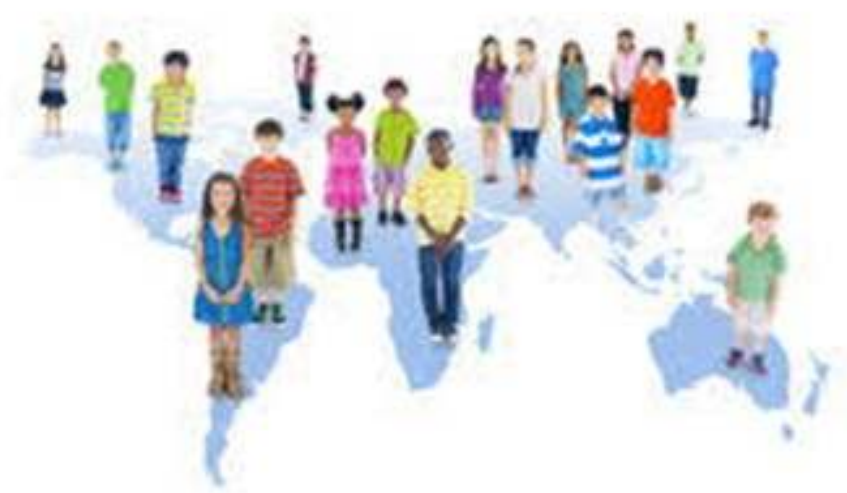

Consigne :

J'observe la photo. Que représente pour toi cette photo ? J'écris des mots en lien avec la photo.

Nous faisons le choix de présenter les résultats de l'analyse à partir d'histogrammes, un outil qui nous semble adéquat au contenu collecté : des mots. Toutefois, il n'est pas question ici d'une analyse quantitative a proprement parlé, ce qui nous intéresse le plus ce sont les idées dégagées des verbatim, dans une approche qualitative compatible avec l'analyse des représentations. Les chiffres présentés se rapportent au nombre du groupe expérimental (30 élèves). 


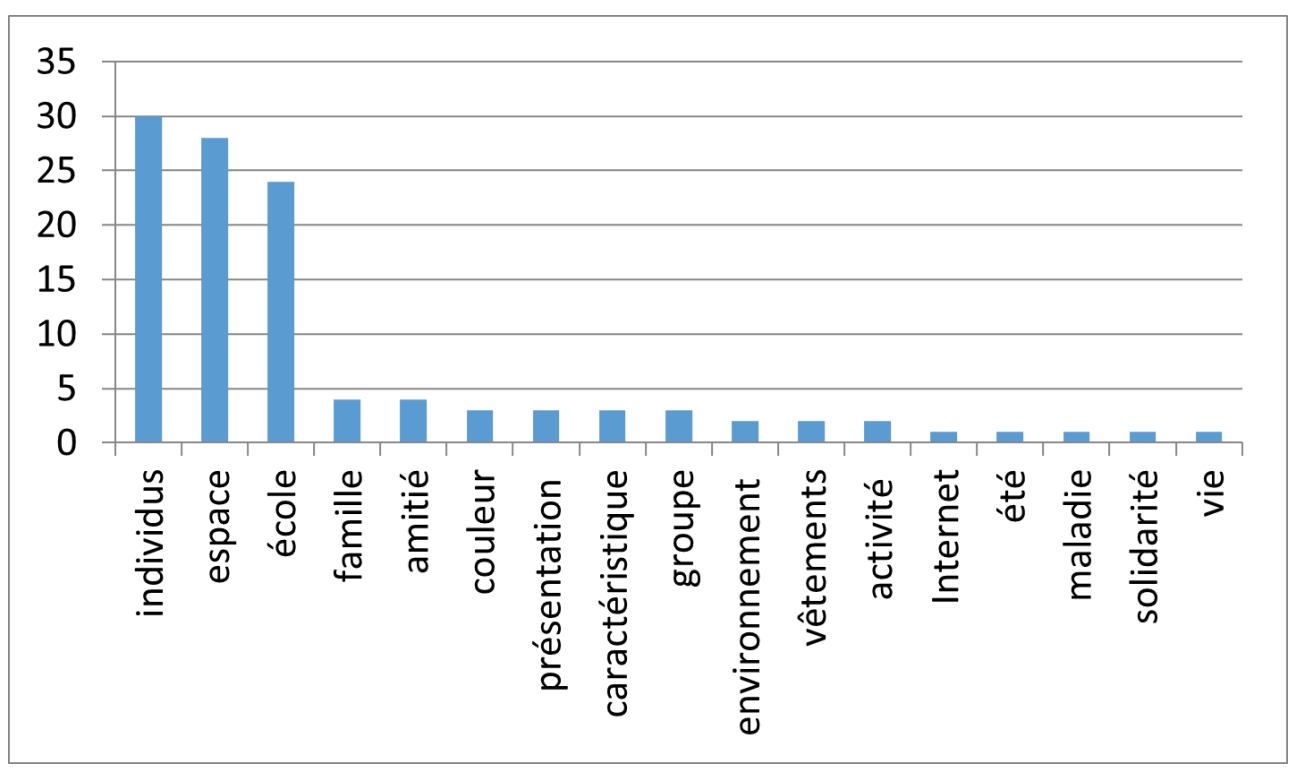

Comme nous pouvons le voir sur ce graphique, tous les apprenants rapprochent l'altérité à des individus : enfants, personnes, hommes, parents. Quelques-uns leur donnent même des caractéristiques: deux voient qu'ils sont gentils, et un dit qu'ils sont méchants. Leurs réponses laissent paraître aussi l'espace géographique: planète, continents, pays. Selon certains, l'image présentée est relative à une institution, en utilisant des mots et expressions telles que : école, classe, camarades, enseignants, saluer le drapeau (la majorité des mots est exprimée en arabe). Trois élèves pensent aux couleurs, différentes couleurs de peau ou de vêtements. Pour d'autres l'image leur rappelle peut-être la famille ou encore les vacances d'été, voire la maladie pour un élève. Seulement quatre d'entre eux pensent à la relation d'amitié, deux à une activité (sport, jouer), probablement en rapport avec les Jeux olympiques. Un à la solidarité et un autre à un espace de rencontre, en l'occurrence Internet.

En plus des deux thèmes explicites sur l'image donnée que sont les individus et les continents, les apprenants infèrent ceux d'union, de plaisir, de rencontre à travers les mots telles que famille, été, jouer, sport.

Beacco (2018 : 231) explique dans ce sens que

«les verbalisations des apprenants sont donc un matériau essentiel à "faire produire", en ce qu'elles sont susceptibles de manifester des attitudes ou des convictions et d'avoir des effets sur celles-ci. ».

À partir du matériau recueilli, nous avons constitué des activités linguistiques et langagières afin de les mettre en pratique pour une expérimentation ayant comme thématique l'altérité qui s'appuient principalement sur l'interaction relationnelle. Où nous avons introduit quelques thèmes dégagés des représentations de notre échantillon que nous synthétisons comme suit

Activités sur l'altérité linguistique et culturelle

Dire bonjour dans différentes langues: Konnichiwa (japonais); i ni sogoma (bambara); Merhaba (turc) ; سلام ou (arabe).

Découvrir et proposer des éléments culturels de certains pays : hockey sur glace (Canada), Kebab (Turquie), Takoyaki (plat japonais), danse Dogon (Mali), la 
mosquée Ketchaoua (à la Casbah d'Alger, Algérie) et d'autres propositions par les apprenants.

Exemple d'activité :

Les correspondants ont écrit bonjour dans leur langue première.

Je complète par la langue qui convient.

Merhaba!

i ni sogoma!

Bonjour!

Konnichiwa!

Activités sur l'altérité conceptuelle :

Connaître le nom des continents et de certains pays / villes à travers des supports géographiques, employer des adjectifs de nationalité, comprendre des expressions de sentiments au moyen d'émoticônes et de photos d'enfants.

Exemple d'activité :

Je complète par des noms de pays.

Raphä̈l habite au

Osman habite en

Soumba habite au

Kaori habite au

Activités portant sur l'altérité communicationnelle relationnelle :

- Rencontrer des enfants du monde à travers des interventions sur Internet simulées (faute de matériel).

- Participer à un échange : se présenter et dire bonjour dans sa langue première.

- Présenter à ses camarades un enfant d'une autre nationalité rencontré lors d'un voyage.

- Dire comment imaginer un monde beau sans pleurs et sans douleurs.

- Écrire pour raconter comment réagir face à une situation de maltraitance d'un réfugié syrien.

\section{Démarche méthodologique :}

La démarche méthodologique adoptée ici s'articule autour de quatre étapes qui sont les suivantes :

mise en situation, qui consiste à mettre les élèves dans une situation de rencontre, d'interaction, d'échange pour donner sens à l'apprentissage ;

phase de compréhension, c'est de commencer par des activités de compréhension (de l'oral et de l'écrit) pour que l'apprenant s'imprègne du lexique relatif à l'altérité et le préparer à la production ;

phase de conceptualisation, qui s'appuie sur une systématisation linguistique afin d'outiller l'apprenant de différentes formes linguistiques l'aidant à produire ;

la phase de production est de mettre l'apprenant dans des situations de production (orale et écrite) pour une expérience active de l'altérité.

Modes d'intervention:

Alterner les modes sémiotiques (graphique, verbal et iconique), ainsi que les canaux (oral et écrit), en veillant à ce qu'il y est un enchaînement logique entre les différentes activités proposées.

- Des interactions réciproques entre les différentes activités proposées sont inévitables.

- Ne pas juger l'avis des apprenants, mais susciter leur curiosité et les amener à partir d'activités adaptées à s'ouvrir à la différence et à la diversité. 
Graphique 2 : Représentations post expérience

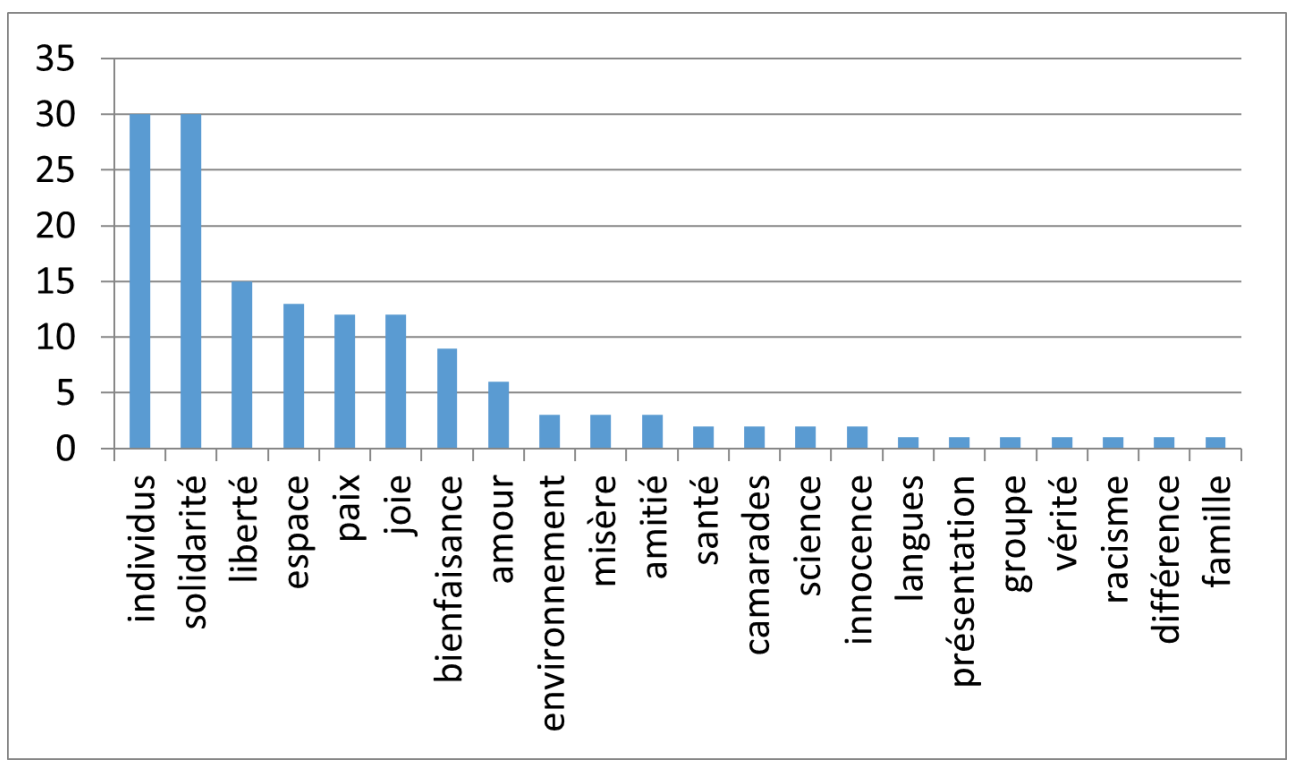

Comme nous pouvons l'observer, après avoir exposé les élèves à différentes activités portant sur l'altérité linguistique et culturelle, conceptuelle, communicationnelle) d'autres thèmes apparaissent dans ce second graphique. Il est question de paix, de joie, de bienfaisance, de science, de langues. En effet, l'ensemble de manières d'être est exprimé à partir d'expressions telles que : paix, monde beau, monde saint, sans guerre, liberté. La notion de joie est représentée par la fête, des enfants heureux, vivre dans la joie, bonheur. Aux yeux de certains apprenants, il faut donner du pain aux faibles, donner des vêtements aux enfants, construire des maisons. D'autres se soucient de l'environnement sur lequel il faut intervenir et arroser les arbres / les fleurs, ramasser les déchets. Neuf enfants expriment l'idée de relation dans les mots amour et amis. Les mots camarades et élèves peuvent également se rapporter au relationnel, en même temps ces mots rappellent le thème de l'école qui figure dans la première présentation.

Par ailleurs, seulement trois élèves pensent à la misère, guerre et tristesse. Un élève emploie l'expression contre le racisme (en arabe). Un parle de vérité, faisant peut-être allusion à la réalité. Nous retrouvons aussi les catégories individus pour l'ensemble, espace pour treize élèves, famille (un seul), solidarité qui revient en force, à égalité avec individus.

En comparant les deux contenus collectés de la même activité Remue-méninges, nous remarquons qu'après avoir exposé les apprenants à une altérité plurielle, leur vision de l'altérité a pris de l'ampleur, passant de dix-sept à vingt-deux thèmes. Et leurs expressions s'élargissent en phrases malgré la précision de la consigne. Cela montre des signes d'ouverture et de respect de l'altérité, notamment par l'expression contre le racisme et le mot différents. Ce qui justifie l'importance de la pluralité dans l'enseignement de l'altérité. D'autre part, la présence de l'identifié dans l'enseignement de l'altérité favorise le sentiment de sécurité indispensable dans l'approche de l'autre.

En somme, les élèves auprès desquels nous avons enquêté démarrent l'expérience avec des représentations de l'altérité plutôt positives, mais leurs expressions montraient un rapport superficiel : personnes, monde, école. À travers leurs réponses, en amont et en post expérience, les apprenants témoignent de l'intérêt vis-à-vis de l'autre. Le nouveau 
et l'inconnu les intéressent et suscitent leur curiosité. Ce qui explique l'apparente évolution des représentations vers l'expression de valeurs universelles liées à l'affect, dans des idées de solidarité; de liberté; de partage; de relationnel façonnés par l'imaginaire des enfants. Ce qui nous permet ainsi de confirmer notre hypothèse selon laquelle exposer les jeunes apprenants à une altérité plurielle en interaction avec l'identité, permet une disposition favorable à l'égard de l'autre.

Tout en gardant à l'esprit que les représentations sont dynamiques et donc susceptibles d'être modifiées, en particulier celles des enfants.

Tout bien considéré, l'interculturel semble en train de basculer vers le rapport à l'altérité. Cette dernière, fortement réclamée dans diverses disciplines, se rapporte, en plus du rapport à l'autre, au rapport à soi. L'éducation à l'altérité suscite l'adhésion véritable aux valeurs par la prise en compte des représentations qui constituent un matériel important pour les professionnels de langues. Les réponses des sujets enquêtés, suite à une exposition à différentes formes d'altérité, laissent paraître des opinions correspondant au savoir-vivre ensemble. Envisager un enseignement/ apprentissage du français dans une dimension plurielle par l'expérience diversifiée de l'altérité, et ce depuis le fondamental est ce qui peut convenir à la complexité de la situation algérienne. Ainsi le résume Gandhi : «il n'est pas nécessaire d'éteindre la lumière de l'autre pour que brille la nôtre ».

\section{BIBLIOGRAPHIE}

Abdelhamid, S. \& Khadraoui, S. (2009). "De la sécurisation de l'interculturalité à l'humanisation de la mondialisation". Synergies Algérie (4), pp. 35-45.

Beacco, J.-C. (2018). L'altérité en classe de langue, Pour une méthodologie éducative. Paris : Didier.

Blanchet, P. (2014). « La dimension interculturelle dans la formations aux langues : et si ça changeait tout ?». [Colloque] https://www.youtube.com/watch ?v =LnW97xyCms0\&t =5s.

Boyer, C. (2000). "Conceptualisation et actions didactiques à propos de la reproduction végétale". Aster [En ligne] . https://hal.univ-lille3.fr/hal-01588436.

Brainçon, M. (2012). L'Altérité enseigante. D'un penser sur l'autre à l'Autre de la penser. Paris : Éditions Publibook.

Briançon, M. (2019). Le sens de l'altérité en éducation : Enjeux, formes, processus, pensées et transferts. Londre : ISTE Editions.

Commision Nationale des Programmes (2016). Enseignement primaire. Programme de la langue française. Ministère de l'Éducation nationale. Direction de l'enseignement fondamental.

Coste, D. \& Cavali, M. (2015). Education, mobilité, altérité. Les fonctions de médiation de l'école. Conseil de l'Europe.

Dakhia, A. (2008). "Désir d'interculturalité : De cette autre idéal humain au devoir de partage". Synergie Algérie (2), pp. 147-154. 
Demorgon, J. (2010). "Comptes rendus de Lecture". Synergies Algérie (11), pp. 233-239.

Djedid, I. \& Aouadi, S. (2015). "L'enfer, est-ce vraiment les autres ? Les représentations de l'altérité culturelleen classe de langue". Synergie Algérie (22), pp. 73-86.

Gaouaou, M. (2009). "L'enseignement/apprentissage du français langue étrangère et la quête d'une nouvelle approche avec les autres cultures". Synergie Algérie (4), pp. 209-216.

Hamidou, N. (2007). "La langue et la culture : une relation dyadique". Synergies Algérie (1), pp. 29-40.

Hamidou, N. (2014). "La dimension interculturelle dans l'enseignement/apprentissage du français en Algérie entre représentations et connaissances culturelles". Multilinguales (3), pp. 125-138.

Jodelet, D. (2005). "Formes et figures de l'altérité" . Dans M. Sanchez-Mazas \& L. Licata, L'Autre : Regards psychosociaux (pp. 23-47). Grenoble : Les Presses de l'Université de Grenoble.

Kordes, H. (1999). "Les expériences d'étrangeté". Dans J. Demorgon, \& E.-M. Lipiansky, Guide de l'interculturel en formation. Paris : Retz.

Lipiansky, E.-M. (1992). "Les théories de la communication - La communication interculturelle". Cahiers français (258).

Merazga, R. (2015). "L'université algérienne à l'ère de l'interculturel : pour une éducation à l'altérité en classe de FLE". Revue de la faculté des lettres et des langues (17), pp. 71-87.

Negura, L. (2006). "L'analyse de contenu dans l'étude des représentations sociales". Sociologies pratiques (1), pp. 1-21.

Piaget, J. (1926). "Les problèmes et les méthodes". Dans La représentation du monde chez l'enfant. Alcan.

Sallabery, J.-C. (1996). Dynamique des représentations dans la formation. L'Harmattan.

\section{NOTES}

1. Proposition d'une forme de néologisme selon laquelle l'interaction, le contact, la diversité, la différence, la pluralité, le respect, la rencontre deviennent source et condition de vie.

2. Source d'entente, de fertilité et de fécondité.

3. Dans un rapport de communication sur la légitimité d'une formation professionnelle à l'altérité. https://icademie-labs.sciencesconf.org/conference/icademie-labs/pages/ Legitimite_d_une_formation_professionnelle_a_l_alterite_Briancon_.pdf.

\section{RÉSUMÉS}

Les nouvelles visions pluralistes inscrivent la didactique de contact dans une dimension relationnelle, favorisant le développement d'attitudes et la formation de la personnalité. Les représentations présentent un répertoire enrichissant pour la conception d'activités sur l'altérité. Par l'expression de ses sentiments, l'enfant modèle sa pensée mêlée d'action et 
d'imaginaire. Dans le cadre d'une expérience exploratoire que nous avons menée auprès d'élèves du primaire, les réponses des sujets montrent de l'intérêt vis-à-vis de l'autre. Par ailleurs, exposer les élèves à différentes formes d'altérité favorise leur rapport à celle-ci, manifestant des attitudes d'ouverture et de respect de l'autre.

The new pluralistic visions place didactics of contact in a relational dimension, favoring the development of attitudes and the formation of personality. Representations are an enriching repertoire for designing activities on otherness. By the expression of his feelings, the child models his thought mixed with action and imagination. As part of an exploratory experience that we conducted with elementary school pupils, the subjects' responses showed interest in the other. In addition, exposing students to different forms of otherness promotes their relationship with it, showing attitudes of openness and respect for others.

\section{INDEX}

Mots-clés : interculturel, rapport à l'altérité, représentations, valeurs

Keywords : intercultural, relationship to otherness, representations, values

\section{AUTEURS}

\section{HIDAYA DJOUDER}

Université Badji Mokhtar, Annaba, Algérie. Laboratoire interdisciplinaire de pédagogie et de didactique (LIPED)

\section{BILLEL OUHAIBIA}

Université Badji Mokhtar, Annaba, Algérie. Laboratoire interdisciplinaire de pédagogie et de didactique (LIPED) 